\title{
Chemotherapy-driven increases in the CDKN1A/PTN/PTPRZ1 axis promote chemoresistance by activating the NF-KB pathway in breast cancer cells
}

Peng Huang ${ }^{1,2}$, Deng-jie Ouyang ${ }^{1}$, Shi Chang ${ }^{2}$, Mo-yun Li ${ }^{1}$, Lun Li ${ }^{1}$, Qian-ying Li ${ }^{1}$, Rong Zeng ${ }^{1}$, Qiong-yan Zou ${ }^{1}$, Juan $\mathrm{Su}^{1}$, Piao Zhao ${ }^{1}$, Lei Pei ${ }^{1}$ and Wen-jun $\mathrm{Yi}^{{ }^{*}}{ }^{*}$

\begin{abstract}
Background: Chemotherapy is the primary established systemic treatment for patients with breast cancer, especially those with the triple-negative subtype. Simultaneously, the resistance of triple-negative breast cancer (TNBC) to chemotherapy remains a major clinical problem. Our previous study demonstrated that the expression levels of PTN and its receptor PTPRZ1 were upregulated in recurrent TNBC tissue after chemotherapy, and this increase was closely related to poor prognosis in those patients. However, the mechanism and function of chemotherapy-driven increases in PTN/PTPRZ1 expression are still unclear.
\end{abstract}

Methods: We compared the expression of PTN and PTPRZ1 between normal breast and cancer tissues as well as before and after chemotherapy in cancer tissue using the microarray analysis data from the GEPIA database and GEO database. The role of chemotherapy-driven increases in PTN/PTPRZ1 expression was examined with a CCK-8 assay, colony formation efficiency assay and apoptosis analysis with TNBC cells. The potential upstream pathways involved in the chemotherapy-driven increases in PTN/PTPRZ1 expression in TNBC cells were explored using microarray analysis, and the downstream mechanism was dissected with siRNA.

Results: We demonstrated that the expression of PTN and PTPRZ1 was upregulated by chemotherapy, and this change in expression decreased chemosensitivity by promoting tumour proliferation and inhibiting apoptosis. CDKN1A was the critical switch that regulated the expression of PTN/PTPRZ1 in TNBC cells receiving chemotherapy. We further demonstrated that the mechanism of chemoresistance by chemotherapy-driven increases in the CDKN1A/PTN/PTPRZ1 axis depended on the NF-kB pathway.

Conclusions: Our studies indicated that chemotherapy-driven increases in the CDKN1A/PTN/PTPRZ1 axis play a critical role in chemoresistance, which suggests a novel strategy to enhance chemosensitivity in breast cancer cells, especially in those of the triple-negative subtype.

Keywords: Breast cancer, Protein tyrosine phosphatase receptor Z1, Pleiotrophin, Chemotherapy resistance, NF-kB signalling pathway

\footnotetext{
* Correspondence: yiwenjun@csu.edu.cn

${ }^{1}$ Department of General Surgery, the Second Xiangya Hospital, Central South

University, No.139 Renmin Road, Changsha 410011, China

Full list of author information is available at the end of the article
}

(c) The Author(s). 2018 Open Access This article is distributed under the terms of the Creative Commons Attribution 4.0 International License (http://creativecommons.org/licenses/by/4.0/), which permits unrestricted use, distribution, and reproduction in any medium, provided you give appropriate credit to the original author(s) and the source, provide a link to the Creative Commons license, and indicate if changes were made. The Creative Commons Public Domain Dedication waiver (http://creativecommons.org/publicdomain/zero/1.0/) applies to the data made available in this article, unless otherwise stated. 


\section{Background}

Breast cancer is the most common malignancy in women and a leading cause of cancer-related death worldwide, while triple-negative breast cancer (TNBC) is a subtype of breast cancer that typically has a relatively poorer outcome compared with other breast cancer subtypes [1, 2]. The TNBC classification applies to all tumours that lack the expression of the endocrine receptors for oestrogen and progesterone (ER and PgR, respectively) and the aberrant expression of HER2. In addition, TNBC typically has an inherently aggressive clinical behaviour and lacks recognized molecular targets for therapy [3]. Nevertheless, chemotherapy is still the most common treatment option for patients with early-stage or advanced-stage TNBC [4]. Approximately $30 \%$ of patients with high-grade TNBC have a strong initial response to neoadjuvant chemotherapy (NAC) and achieve a pathologic complete response (pCR); however, the resistance of TNBC to chemotherapy remains a major clinical problem since approximately $20 \%$ of patients with TNBC exhibit progression during NAC or shortly after therapy due to drug resistance [5]. Therefore, understanding the molecular basis of chemotherapy and identifying novel molecular targets are essential to improving chemotherapy efficacy in patients with TNBC.

Recently, multiple lines of evidence suggested that the expression of pleiotrophin (PTN), which is a secreted growth factor that binds to receptor protein tyrosine phosphatase zeta (PTPRZ1) to stimulate human endothelial cell migration, is associated with poor prognosis in a variety of malignant tumours [6]. Our previous study found that variation in the expression of PTPRZ1 was observed between recurrent TNBC tissue and nonrecurrent TNBC tissue, which indicated that PTPRZ1 may be a novel risk factor for poor prognosis in TNBC [7]. However, the precise mechanisms whereby PTN/ PTPRZ1 signalling regulates the chemotherapy sensitivity of TNBC cells are not well understood.

Our current studies showed that the expression of PTN and its receptor PTPRZ1 in human breast cancer tissue depended on whether the patient received chemotherapy, and chemotherapy-driven increases in PTN/PTPRZ1 signalling could inhibit chemotherapy responsiveness in TNBC cells. In the current work, mechanistic and functional studies were performed to thoroughly elucidate the molecular players involved in the PTN/PTPRZ1 signalling that regulates chemoresistance and to determine the potential roles of PTN/PTPRZ1 in chemotherapy resistance.

\section{Methods}

\section{Microarray analysis of data from the GEPIA database and GEO database}

To investigate the expression levels of the same gene in breast cancer and normal tissues, we performed an analysis using the GEPIA database (Gene Expression Profiling
Interactive Analysis, http://gepia.cancer-pku.cn/index.html) [8], which contains 1085 breast cancer tissue samples and 291 normal tissue samples. To compare the expression levels of the same gene in breast cancer tissue before and after chemotherapy, we analysed a cohort from the GEO database (GSE87455, https://www.ncbi.nlm.nih.gov/geo/) that included 69 samples from breast cancer patients with gene expression data (pretreatment/posttreatment).

\section{Cell culture}

The breast cancer cell lines HCC-1937, MCF-7, MCF-7/ADR, MDA-MB-231 and MDA-MB-453 were purchased from the Cell Bank of the Chinese Academy of Sciences (Shanghai, China). The cells were grown in $25 \mathrm{~cm}^{2}$ cell culture flasks and maintained in Dulbecco's-modified Eagle's medium (DMEM, HyClone) or RPMI 1640 medium (HyClone) supplemented with $10 \%$ foetal bovine serum, $100 \mathrm{U} / \mathrm{ml}$ penicillin $\mathrm{G}$, and $100 \mathrm{U} / \mathrm{ml}$ streptomycin at $37{ }^{\circ} \mathrm{C}$ in $5 \% \mathrm{CO} 2$ and $95 \%$ air as previously described [9]. Dox was purchased from Yuanye Biotech (Shanghai, China) and diluted in PBS. rhPTN was purchased from PeproTech (London, UK), and cells were treated with rhPTN $(5 \mu \mathrm{g} / \mathrm{mL})$ for $20 \mathrm{~min}$ according to the manufacturer's instructions.

\section{Immunofluorescent staining}

Cells were washed with PBS twice and then fixed with $4 \%$ paraformaldehyde. The cells were incubated with anti-PTPRZ1 primary antibodies (BD Biosciences, 1:200) overnight at $4{ }^{\circ} \mathrm{C}$. Cells were washed with PBS and then incubated with the appropriate conjugated secondary antibodies (Sangon Biotech, Shanghai, China, 1:500) for $1 \mathrm{~h}$ at $37^{\circ} \mathrm{C}$. Finally, the cells were stained with DAPI $(50 \mu \mathrm{g} / \mathrm{ml})$ for $20 \mathrm{~min}$ for nuclear imaging. At least 100 cells per well were counted in 3 independent experiments.

\section{Quantitative RT-PCR}

Total RNA was extracted from breast cancer cells using Trizol (Invitrogen, Carlsbad, CA). As described in our previous work [9], qRT-PCR was performed on a Prism ${ }^{\circ}$ 7300 Real-time PCR System (Applied Biosystems, Foster City, CA, USA) using a $\mathrm{SYBR}^{\circ}$ Green PCR master mix (Applied Biosystems, Foster City, CA, USA). After the reactions were completed, the comparative threshold cycle $(\mathrm{Ct})$ method was used to calculate the relative gene (e.g., PTN, PTPRZ1, IKK $\alpha, I K K \beta, I \kappa B \alpha, ~ p 65$ and p50) expression. U6 expression was used as the internal control. Human-specific primers were synthesized, and their sequences are shown in Table 1.

\section{Cell transfection}

The PTPRZ1-overexpression plasmid and CDKN1Aoverexpression plasmid were obtained from Professor Jun-li Luo (Laboratory of Gene Regulation and Signal 
Table 1 Primers designed for RT-PCR

\begin{tabular}{|c|c|c|}
\hline \multicolumn{2}{|l|}{ Gene } & \multirow{2}{*}{$\begin{array}{l}\text { Sequence } \\
\text { TGGGAAAACAGTGGAAAT }\end{array}$} \\
\hline PTPRZ1 & Sense & \\
\hline & Antisense & CCGCATCAAAGCAGTAGA \\
\hline \multirow[t]{2}{*}{$\beta$-actin } & Sense & AGGGGCCGGACTCGTCATACT \\
\hline & Antisense & GGCGGCACCACCATGTACCCT \\
\hline \multirow[t]{2}{*}{ IKKa } & Sense & TTGTAGTITAGTAGTAGAACCCAT \\
\hline & Antisense & ATTCCAGTTTCACGCTCA \\
\hline \multirow[t]{2}{*}{ IKK $\beta$} & Sense & TGAATGAGGATGAGAAGACTG \\
\hline & Antisense & GACCACGGACCTTGCTAC \\
\hline \multirow[t]{2}{*}{ IKBa } & Sense & GCAGCAGACTCCACTCCAC \\
\hline & Antisense & TCCACGATGCCCAGGTAG \\
\hline \multirow[t]{2}{*}{ p65 } & Sense & GGCCATGGACGAACTGTT \\
\hline & Anti-sense & GGTCTTGGTGGTATCTGTGCT \\
\hline \multirow[t]{2}{*}{ p50 } & Sense & GTCTTACCCTCAGGTCAAAA \\
\hline & Antisense & TGTCATTCGTGCTTCCAG \\
\hline
\end{tabular}

Transduction, Department of Pharmacology and Cancer Center, School of Medicine, UCSD, La Jolla, California, USA) as gifts, and the knockdown plasmids were constructed by GenePharma (GenePharma Co., Ltd., Shanghai, China). The sequences of siPTPRZ1 (sense: 5' -AAAUGCGAAUCCUAAAGCGUU-3'; antisense: 5' -A ACGCUUUAGGAUUCGCAUUU-3'), siPTN (sense: 5' $-\mathrm{C}$ CAGCAAUAUCAGCAGCAATT; antisense: $5^{\prime}$-UUGCUG CUGAUAUUGCUGGTT), and siCDKN1A (sense: 5'-GAU GGAACUUCGACUUUGUTT-3'; antisense: 5'ACAAAG UCGAAGUUCCAUCTT-3') were the same as those in previous reports $[10,11]$. Lipofectamine and Plus reagent (Invitrogen) was used for transfection following the manufacturer's instructions. After incubation with the transfection mixture for $6-8 \mathrm{~h}$, cells were cultured with fresh medium containing $10 \%$ FBS for the following experiments.

\section{Western blot analysis}

Nuclear and cytoplasmic proteins were fractionated as previously described [12]. The quantification of the protein extract was carried out using a BCA protein quantitation kit (P001B, Auragene, Changsha, China). The protein samples $(25 \mu \mathrm{g})$ were fractionated on $8 \%$ SDSpolyacrylamide gels and transferred onto pure nitrocellulose membranes (Sangon Biotech, Shanghai, China). The membranes were then probed with primary antibodies, and GAPDH and $\mathrm{H} 1$ were used as internal controls. The following primary antibodies were used for western blotting: anti-PTN (Abcam, \#ab79411, 1:10000); anti-PTPRZ1 (Abcam, \#ab126497, 1:1000); anti-CDKN1A (Santa Cruz, \#sc-817, 1:1000); anti-p-IKK $\alpha / \beta$ (Ser176/180) (Cell Signaling, \#2697S, 1:1000); anti-IKK $\alpha$ (Cell Signaling, \#2682S, 1:1000); anti-IKK $\beta$ (Cell Signaling, \#2684S, 1:1000); anti-

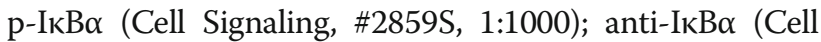

Signaling, \#9242S, 1:1000); anti-p-p65 (Ser536) (Cell Signaling, \#3031S, 1:1000); anti-p65 (Cell Signaling, \#8242S, 1:1000); anti-H1 (Santa Cruz, \#sc-8030, 1:1000); and anti-GAPDH (Abcam, \#ab9485, 1:2500).

\section{Colony formation efficiency assay}

After treatment, MDA-MB-231 cells were plated in a six-well plate at a density of 300 cells/well and the six-well plate was incubated at $37^{\circ} \mathrm{C}$ in a humidified incubator for 10 days. The cell colonies were stained with $0.5 \%$ crystal violet solution (Solarbio, Beijing, China), and the colonies were counted.

\section{Cell proliferation assay}

The in vitro growth of cells treated with Dox (Yuanye Biotech, Shanghai, China) or Cisplatin (DDP, Yuanye Biotech, Shanghai, China) was detected with a CCK8 assay kit (Sangon Biotech, Shanghai, China) according to the manufacturer's instructions. The cell growth curve was generated from the corresponding normalized OD450 values.

\section{Flow cytometry analysis}

The cells for the apoptosis analysis were stained with anti-Annexin $\mathrm{V}$ antibody and propidium iodide using an Annexin V-FITC Apoptosis Detection kit (Sangon Biotech, Shanghai, China), and the percentage of apoptotic cells was examined with flow cytometry (BD Bioscience, CA, USA).

\section{Microarray analysis}

Microarray analysis was conducted to investigate the expression changes of chemotherapy-driven genes. MDA-MB-231 cells were treated with Dox $(1 \mu \mathrm{g} / \mathrm{ml})$ or PBS for $24 \mathrm{~h}$ in triplicate, and then equal quantities of the cells from each triplicate were mixed to generate one sample for each group. Microarray-based mRNA expression profiling was carried out at Genenergy Biotech (Shanghai, China).

\section{Statistical analyses}

All data were included for statistical analyses performed with SPSS 22.0 or GraphPad Prism 6.0 software. An unpaired Student's t-test (two-tailed) was used for the comparison between two unpaired groups, and one-way ANOVA was applied for multi-group data comparison. Bar graphs are presented as the mean \pm s.e.m., with the level for statistical significance set to $* p<0.05$ or $* * 0<01$.

\section{Results}

The expression of PTN and its receptor PTPRZ1 were upregulated in human breast cancer tissue after chemotherapy

To assess the expression of PTN and PTPRZ1 in breast cancer and their relationship with chemotherapy, we obtained RNA sequencing expression data for 291 normal 
breast tissue samples and 1085 cancer tissue samples from the GEPIA database (http://gepia.cancer-pku.cn/index.html); none of the patients who donated the samples were treated with chemotherapy; additionally, we downloaded matched before and after chemotherapy gene expression data for breast cancer tissue samples from 69 patients from the GEO database (GSE87455) (https://www.ncbi.nlm.nih.gov/geo/). The expression levels of both PTN and PTPRZ1 were significantly downregulated in the breast cancer group compared to the normal group $(p<0.05)$ (Fig. 1a, b), and there was no significant correlation between the expression levels of PTN and PTPRZ1 $\left(p>0.05, R^{2}=0.00048\right)$ (Fig. 1c). Interestingly, the expression levels of PTN and PTPRZ1 were both upregulated after chemotherapy (Fig. 1d, e), and a significantly positive correlation between the expression levels of the two proteins was also observed $\left(p<0.05, R^{2}=0.5393\right)$ (Fig. 1f).

\section{Doxorubicin upregulated the expression of PTN and} PTPRZ1 and formed a positive feedback loop in TNBC cells We then detected the expression of PTN and PTPRZ1 in different breast cancer cell lines via RT-PCR, and we found that PTN and PTPRZ1 were expressed at higher levels in the MDA-MB-231 and MDA-MB-453 TNBC cells than the other breast cancer cells, HCC-1937 and MCF-7. We also found that the expression levels of PTN and PTPRZ1 were significantly increased in chemotherapy-resistant cell lines (MCF-7/ADR) compared with those of the chemotherapy-sensitive cell line (MCF-7) (Fig. 2a, b). Dox treatment increased the expression of PTN and PTPRZ1 in MDA-MB-231 TNBC cells in a dose-dependent manner (Fig. 2c). Additionally, in MDA-MB-231 cells, we found that Dox and PTN are two independent factors that promote the upregulation of PTPRZ1 expression (Fig. 2d, h). To address whether PTPRZ1 also affected the expression of PTN, we investigated whether knockdown or increasing PTPRZ1 expression in MDA-MB-231 cells could attenuate or enhance the Dox-driven PTN upregulation. We found that decreased PTPRZ1 expression could significantly offset the Dox-driven increase in the PTN level in MDA-MB-231 cells; conversely, when the expression of PTPRZ1 increased, the Dox-driven PTN level correspondingly increased (Fig. 2f). These data definitively
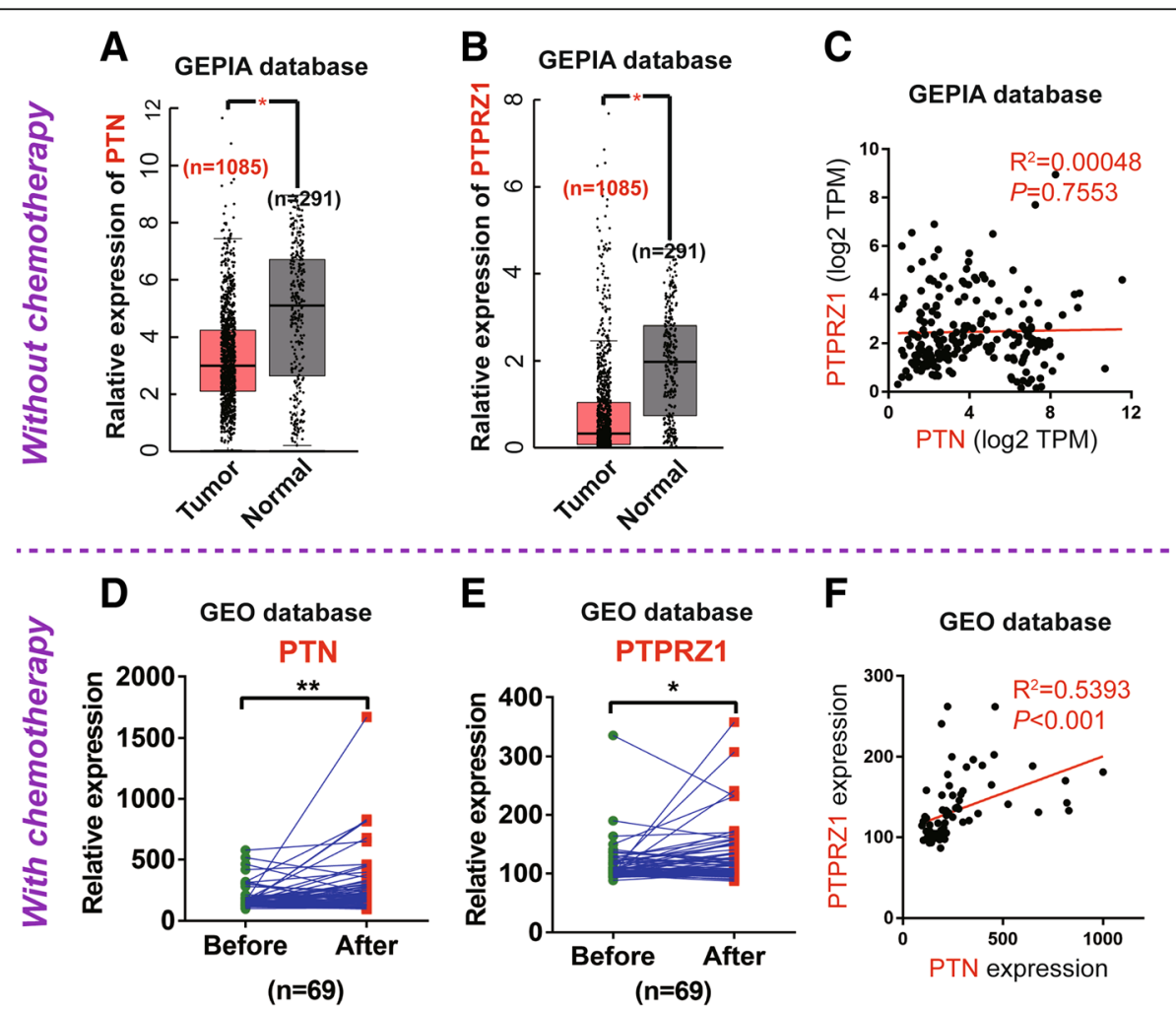

Fig. 1 The expression levels of PTN and PTPRZ1 were increased in breast cancer tissue after chemotherapy. PTN (a) and PTPRZ1 (b) expression data in human normal breast tissue $(n=291)$ and breast cancer tissue $(n=1085)$ from breast cancer patients without chemotherapy were obtained from the GEPIA database (http://gepia.cancer-pku.cn/index.html), Student's t-test, ${ }^{*} p<0.05$. c The correlation analyses of PTN and PTPRZ1 expression in breast cancer specimens without chemotherapy from the GEPIA database are shown, $\mathrm{n}=1085, p=0.7557$, Pearson's $r$ test. Paired expression data of PTN (d) and PTPRZ1 (e) in human breast cancer specimens before and after adjuvant chemotherapy were obtained from the GEO database (https://www.ncbi.nlm.nih.gov/geo/), $n=69, \mathrm{t}$-test, ${ }^{*} p<0.05,{ }^{* *} p<0.01$. $\mathbf{f}$ The correlation analyses of PTN and PTPRZ1 expression in breast cancer specimens after adjuvant chemotherapy is shown, $n=69, p<0.001$, Pearson's $r$ test 


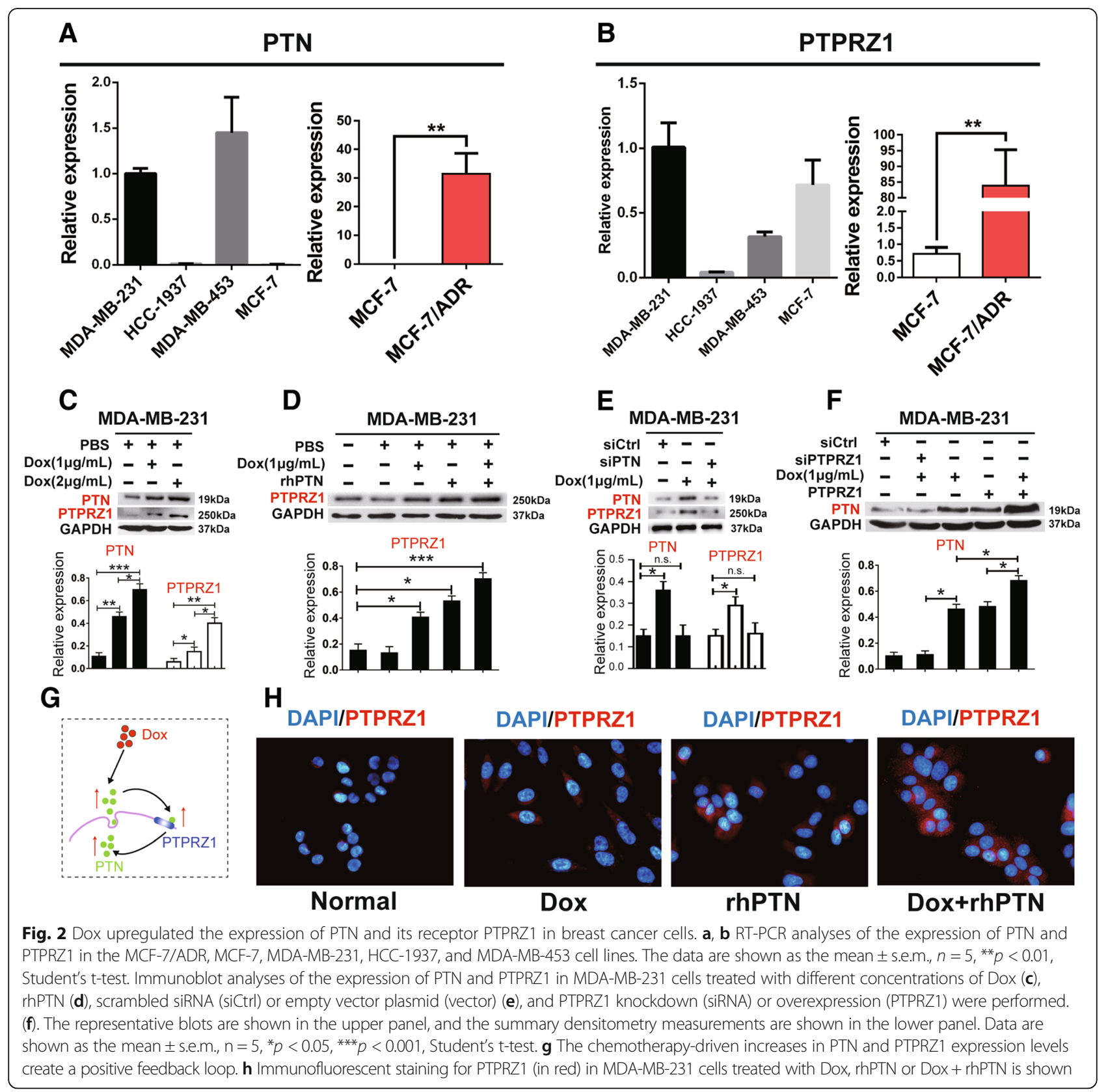

demonstrated that chemotherapy upregulated the expression of PTN and PTPRZ1 in TNBC cells and formed a positive feedback loop between the expression of PTN and that of PTPRZ1 (Fig. 2g).

PTN/PTPRZ1 reduced chemosensitivity by promoting tumour proliferation and inhibiting apoptosis

To understand whether the chemotherapy-driven upregulations of PTN and PTPRZ1 expression levels functioned as tumour protective factors that promote breast cancer cell growth, we investigated whether knockdown or increasing PTN and PTPRZ1 expression could attenuate the effect of chemotherapy in vitro. Our results confirmed that upregulating PTPRZ1 expression or adding rhPTN in the cell culture medium could offset the proliferation inhibition of Dox or DDP, whereas knockdown the expression of PTPRZ1 could enhance the proliferation inhibition of chemotherapy in MDA-MB-231 cells (Fig. 3a, b). Consistently, knockdown of the expression level of PTN or PTPRZ1 significantly increased the inhibition of colony formation (Fig. 3c) and the number of apoptotic cells (Fig. 3d), which correspondingly indicated that siPTN and siPTPRZ1 exerted chemotherapy sensitization effects. Furthermore, we also demonstrated that the inhibition of colony formation and promotion of apoptosis by Dox were rescued when exogenous PTN or endogenous 

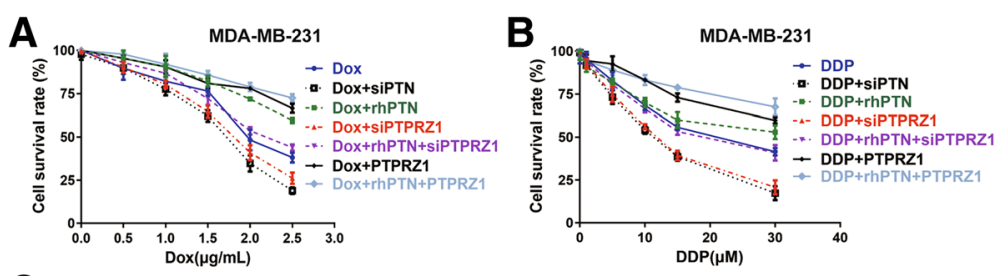

C
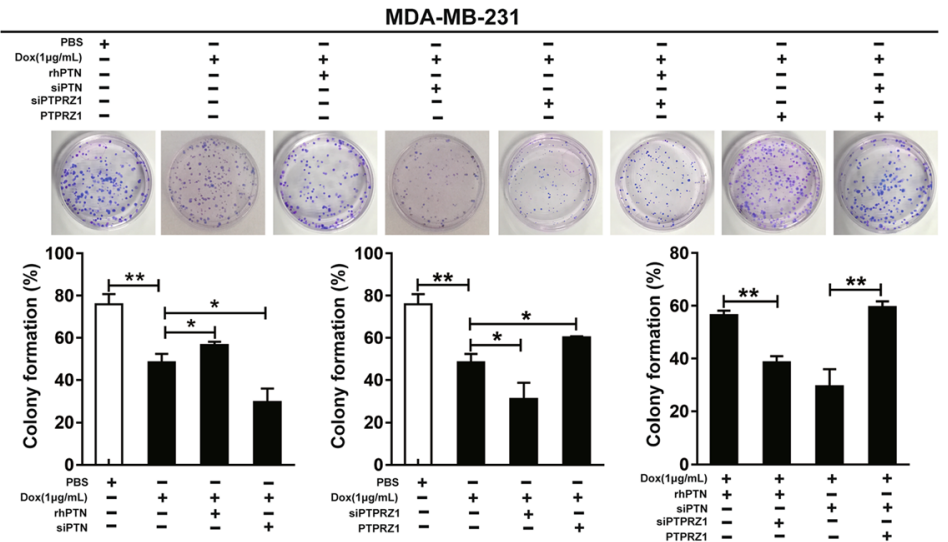

D
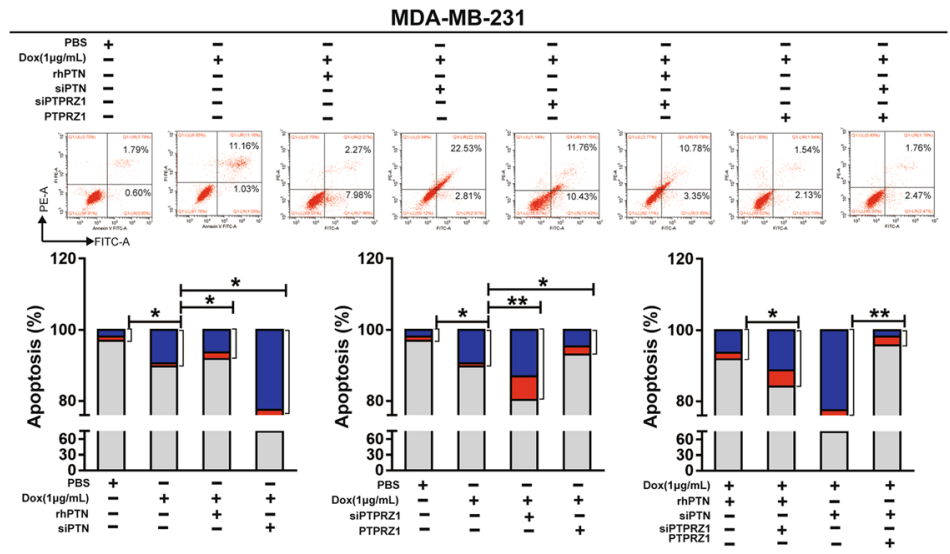

Fig. 3 PTN/PTPRZ1 are involved in breast cancer cell chemoresistance. MDA-MB-231 cells, which were transfected with siPTPRZ1, PTPRZ1, or siPTN to regulate endogenous protein, or added exogenous protein (rhPTN) in the cell culture medium to increase PTN levels, were treated with different concentrations of Dox or DDP. $\mathbf{a}, \mathbf{b}$ Cell viability was determined with a CCK8 assay. $\mathbf{c}$ Cell growth was measured by colony formation assays. The data are shown as the mean \pm s.e.m. of three independent experiments. The statistical significance of the between-group differences was evaluated with a Student's t-test, ${ }^{*} p<0.05$, ${ }^{* *} p<0.01$, n.s. means not significant. $\mathbf{d}$ Apoptosis was measured by flow cytometry based on Annexin V/PI double staining. The data are shown as the mean \pm s.e.m. of three independent experiments. The statistical significance of the between-group differences was evaluated with a Student's t-test, ${ }^{*} p<0.05$, ${ }^{* *} p<0.01$

PTPRZ1 was increased (Fig. 3c and d) in MDAMB-231 cells.

CDKN1A regulated dox-driven PTN secretion in TNBC cells To explore the potential upstream mechanism that regulates chemotherapy-driven PTN/PTPRZ1, we analysed the Dox-affected genes in TNBC cells with a high-throughput microarray. We noticed that CDKN1A (cyclin-dependent kinase inhibitor 1A) ranked first among 60 genes significantly upregulated after Dox treatment (average fold changes> 3.0) relative to the matched negative control in MDA-MB-231 cells (Fig. 4a). When we used the same GEO microarray datasets
(GSE87455), we found that there was a significant correlation between the expression levels of CDKN1A and PTN after chemotherapy $\left(p<0.05, R^{2}=0.6015\right)$, whereas there was no significant correlation before treatment $\left(p>0.05, \mathrm{R}^{2}=0.01788\right.$ ) (Fig. $4 \mathrm{~b}$ ). Interestingly, we found that the expression of CDKN1A was significantly upregulated after chemotherapy (Fig. 4c). To confirm the relationship between CDKN1A and Dox-driven PTN expression or Dox-driven PTPRZ1 expression, we knocked down or increased the expression of CDKN1A in MDA-MB-231 cells treated with Dox. We found that the expression levels of CDKN1A and Dox-driven PTN or Dox-driven PTPRZ1 formed a positive correlation. 


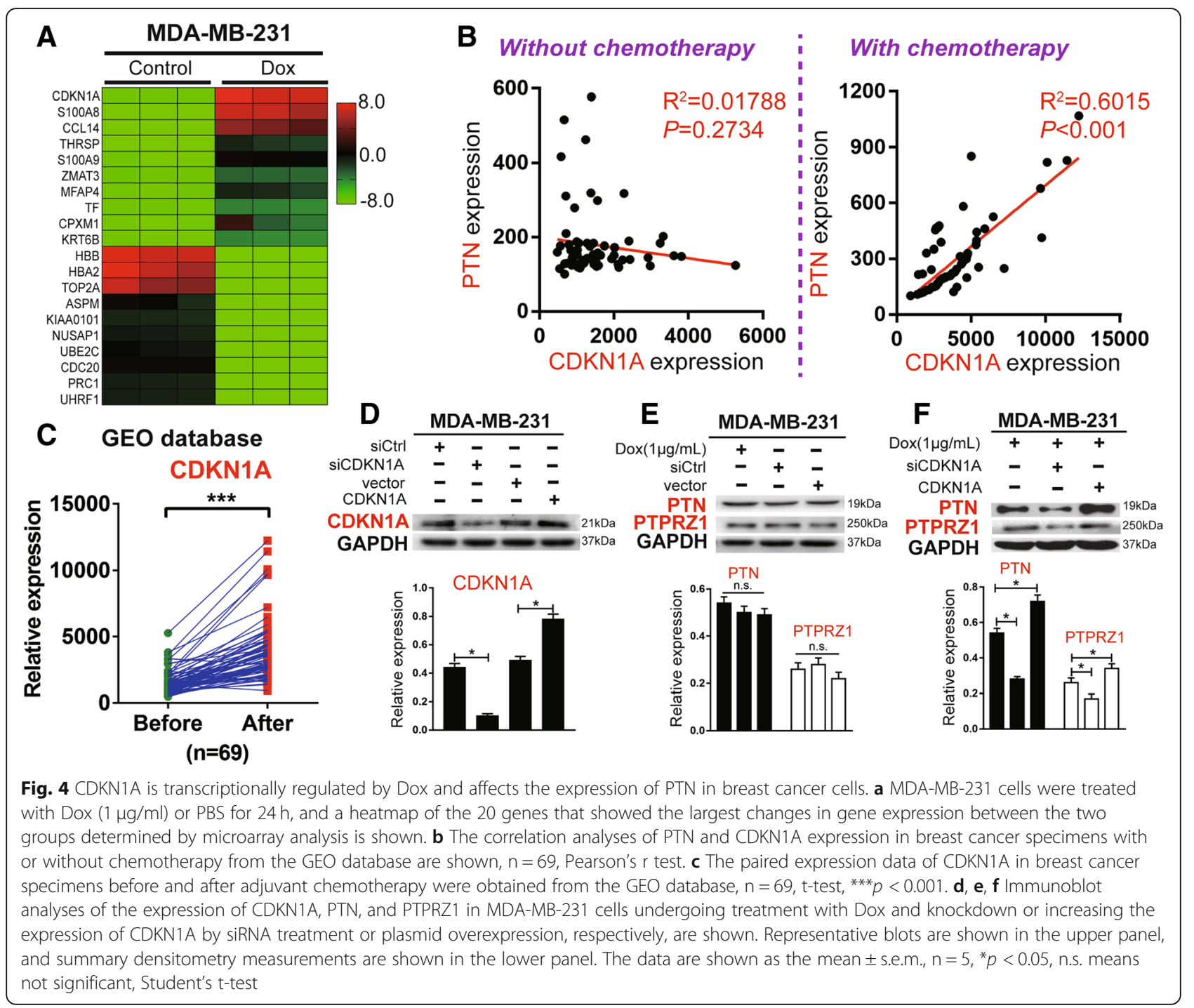

Additionally, the regulatory effect of CDKN1A on PTPRZ1 was not as obvious as the effect on PTN, so we presumed that CDKN1A may directly regulate PTN levels, but the present results were not enough to prove whether CDKN1A directly regulates PTPRZ1 levels (Fig. 4e and f).

\section{Elevated CDKN1A expression correlated with chemoresistance} in TNBC cells

CDKN1A has been described previously as an inhibitor of cyclin-dependent kinase and plays an important regulatory role in cell proliferation, differentiation and senescence, but its function in the development of different cancers is not consistent [13-15]. To investigate whether CDKN1A acts as a tumour protective factor in TNBC during chemotherapy, we evaluated the effect of knocking down or increasing the expression of CDKN1A on colony formation and apoptosis in MDA-MB-231 cells. We demonstrated that the ability to undergo colony formation or apoptosis under Dox treatment was significantly affected by the change in CDKN1A expression, and CDKN1A expression protected MDA-MB-231 cells from inhibition by Dox (Fig. 5a and b).

\section{NF-KB pathway activation by excess PTPRZ1}

Recently, multiple studies have demonstrated that $\mathrm{NF}-\mathrm{kB}$ activation enhances chemoresistance processes [16]. Here, we found that the classical NF- $\kappa B$ signalling pathway was activated in TNBC cells by treatment with Dox. The relative expression levels of NF- $\mathrm{KB}$ pathway components, such as IKK $\alpha$, p65 and p50, were upregulated, while the relative expression levels of IKK $\beta$ and I $\mathrm{K} \alpha$ were downregulated according to an analysis using RT-PCR (Fig. 6a). We also demonstrated that the gene expression pattern of the Dox + PTPRZ1 group was similar to that of the Dox group, but the relative expression levels of the genes of the NF- $\mathrm{B}$ B signalling pathway 


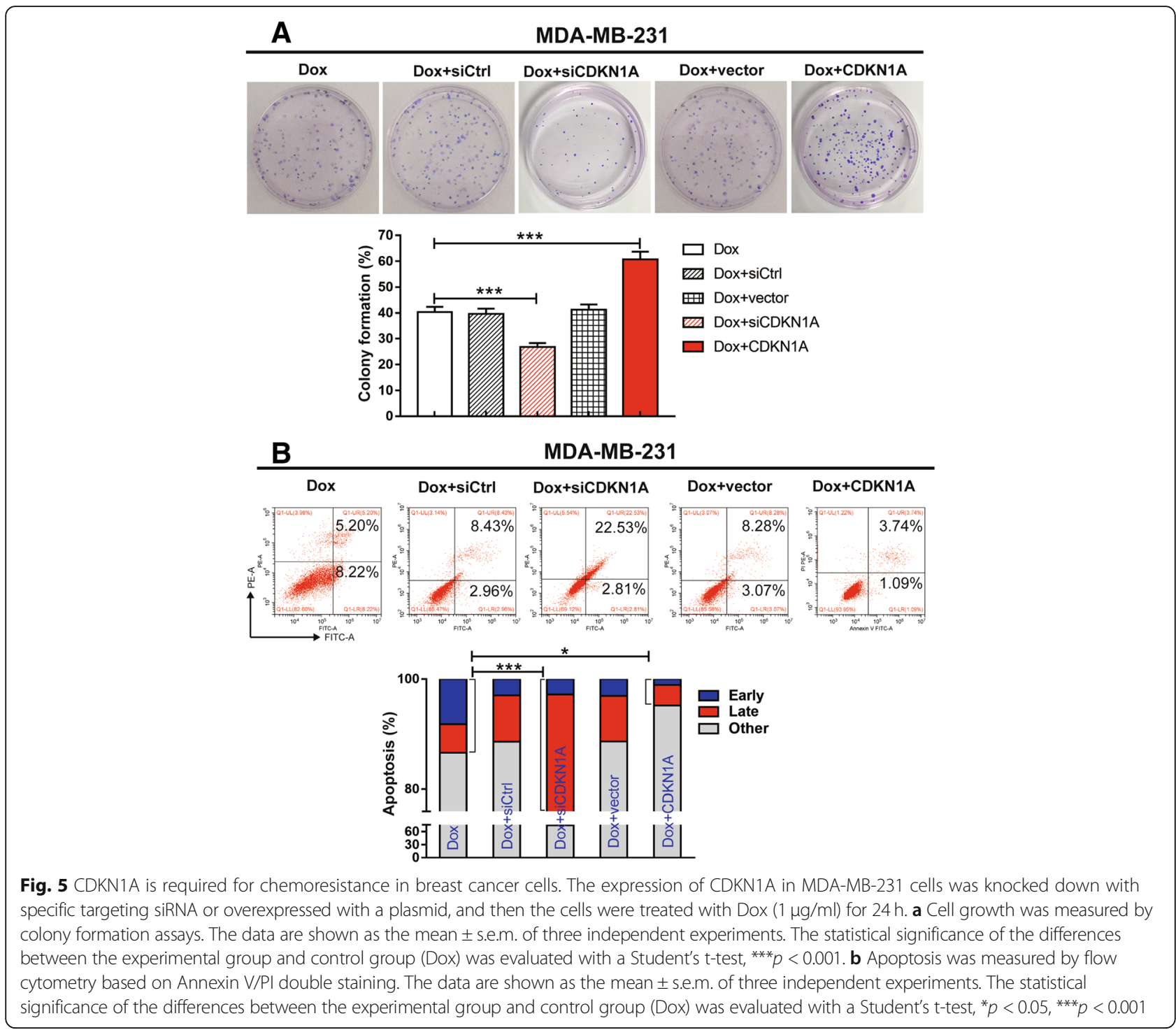

were significantly higher or lower in the Dox + PTPRZ1 group compared to the vector control group (Fig. 6a). To obtain further proof of NF- $\mathrm{kB}$ activation, the phosphorylation of NF- $\mathrm{kB}$ pathway components was also detected by western blotting. We found that the levels of phosphorylated NF-kB pathway components, such as $\mathrm{p}-\mathrm{IKK} \alpha / \beta$ (Ser176), p-IкB $\alpha$ (Ser32) and p-p65(Ser536), were increased after NF- $\mathrm{kB}$ activation, while non-phosphorylated NF- $\mathrm{kB}$ components were unaffected or showed a trend towards reduced levels (Fig. 6b). Therefore, our results strongly suggested that chemotherapy-driven PTPRZ1 expression enhanced the activation of the NF- $\mathrm{kB}$ signalling pathway during chemoresistance processes in TNBC cells (Fig. 6c).

\section{Discussion}

Chemotherapy plays an important role in breast cancer management, and one of the main barriers to cure breast cancer is the chemoresistance, both intrinsic and therapy-induced, of breast cancer, especially the TNBC subtype [17]. To overcome this obstacle, it is important to identify the critical determinants of chemoresistance and to develop safe and effective tumour chemosensitizers. Recently, emerging evidence has demonstrated that PTN/PTPRZ1 signalling might be involved in the carcinogenesis of several cancers; PTPRZ1 interacts with its ligand PTN to mediate tumour cell growth and metastasis [18]. It has been demonstrated that PTPRZ1 binding to PTN could activate calmodulin to induce nitric oxide formation, which causes tumour blood vessel formation and tumour cell proliferation in vitro [19]. Moreover, downregulating PTPRZ1 expression in glioblastoma multiforme (GBM) cells produced a decrease in tumour migration and proliferation [18]. Similar results have also been documented in the carcinogenesis of breast cancer. 


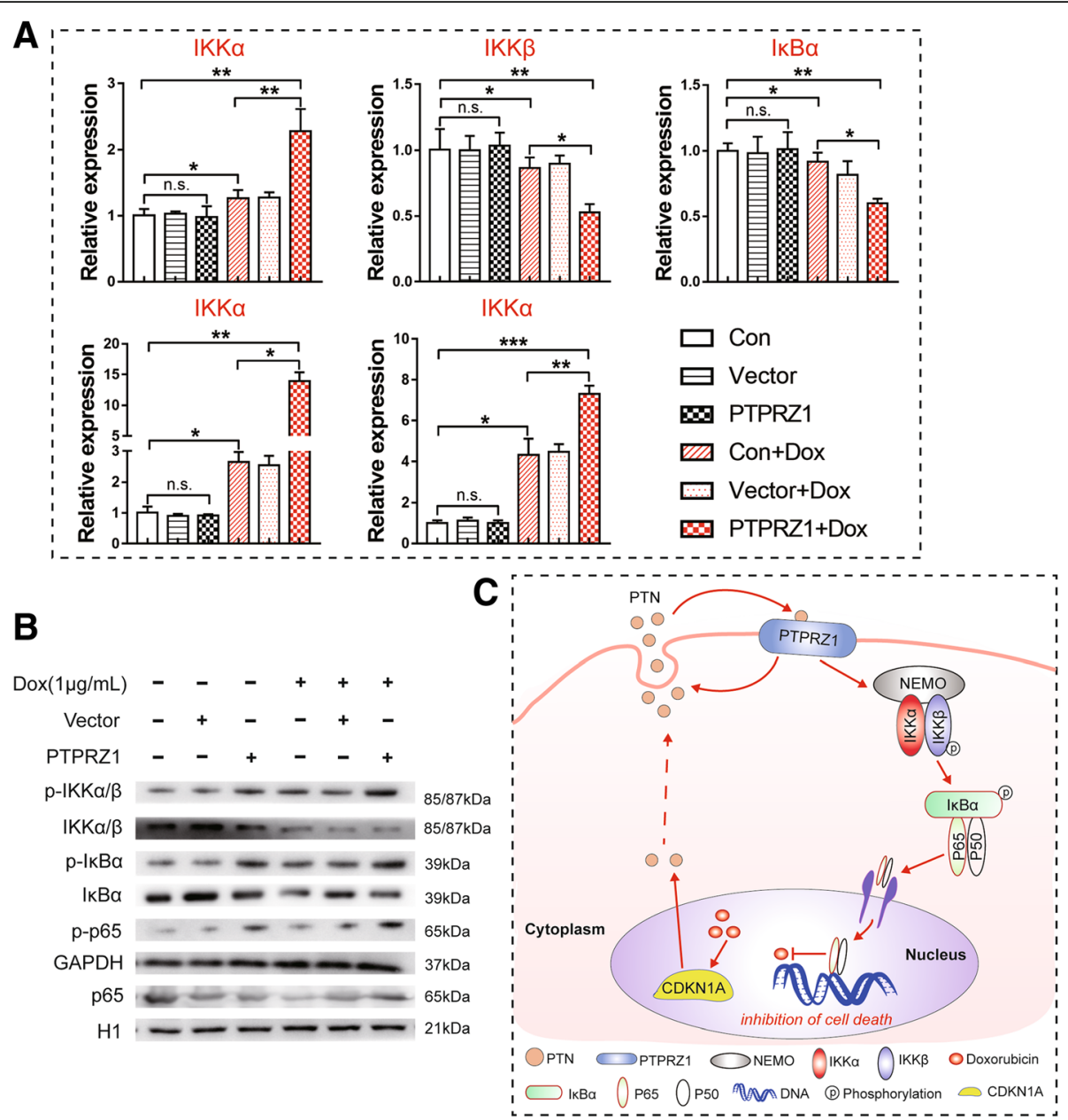

Fig. 6 PTPRZ1 activated the NF-KB pathway in breast cancer cells following treatment with Dox. a RT-PCR analyses of the expression of NF-KB pathway components, including IKKa, IKKB, IKBa, p65 and p50, in MDA-MB-231 cells transfected with PTPRZ1, siPTPRZ1 or scrambled siRNA (vector) and then treated with Dox $(1 \mu \mathrm{g} / \mathrm{ml})$ for $24 \mathrm{~h}$ are shown, $n=3$, Student's t-test, ${ }^{*} p<0.05,{ }^{* *} p<0.01$, ${ }^{* * *} p<0.001$. b The phosphorylation of NF-KB pathway components was detected by western blotting; NF-KB activation was investigated by assessing the phosphorylation of IKKa/ $\beta$ (Ser176), IKBa (Ser32) and p65(Ser536). c Proposed model for mechanisms of CDKN1A/PTN/PTPRZ1-induced chemoresistance in breast cancer cells

On the one hand, studies have found that a low number of breast cancer cases have detectable expression of PTN, while normal breast tissue does not express PTN, while on the other hand, PTN gene expression could play a role in the invasive and/or metastatic behaviour of cell lines or the original tumours the cell lines were obtained from, and targeting PTN with a dominant negative PTN reversed the malignant phenotype of human breast cancer cells in vivo [20,21].

In recent years, conflicting data on the role of PTN and PTPRZ1 expression in breast cancer was existed in different reports, some studies shown over-expression and others shown decreased expression, what interested us was that over-expression of PTN or PTPRZ1 often related to a more aggressive breast cancer phenotype $[22,23]$. Our previous study demonstrated that PTN and PTPRZ1 were highly expressed in recurrent TNBC tissue, which hinted at their tumour-promoting effects [7]. It is well known recurrent TNBC tissues are that kind of breast cancer resistant to chemotherapy and most of them coming from the aggressive phenotype. That means chemotherapy drugs may be one of the activation factors for PTN/PTPRZ1 pathway, following with an even bad chemotherapy sensitivity to these aggressive breast cancer phenotypes. In this study, we uncovered that the protein levels of PTN and PTPRZ1 are significantly higher in human breast cancer tissue after receiving chemotherapy compared with cancer tissue from the same patient prior to chemotherapy. The disruption of PTN/PTPRZ1 signalling by siRNAs could largely abrogate the therapeutic resistance effects of PTN/PTPRZ1 signalling and largely suppress TNBC cell growth during chemotherapy. It is well confirmed that three PTPRZ isoforms are generated by alternative splicing from a 
single PTPRZ gene: two transmembrane isoforms, PTPRZ-A and PTPRZ-B, and one secretory isoform, PTPRZ-S [24]. Since PTPRZ binds PTN mostly through its glycans, deglycosylation of PTPRZ-B would definitely reduce the affinity of PTPRZ-B for PTN [25]. In this study, only the deglycosylated form of PTPRZ-B was observed in breast cancer cells treated with Dox, and the other two variants, full-length PTPRZ-A and PTPRZ-S, were not detected in breast cancer cells. Although our research is the first to indicate that the therapy-induced chemoresistance of breast cancer is regulated by PTPRZ1, the mechanism behind the decreases in the other splicing variants and deglycosylated isoforms that occur during chemotherapy need further exploration.

To understand the upstream mechanism of chemotherapy-driven increases in PTN expression in breast cancer, we found that the nucleoprotein CDKN1A exhibited the most upregulated expression after treatment with Dox via microarray analysis. We also found that the expression of PTN in breast cancer cells which were undergoing Dox treatment was significantly decreased when CDKN1A was knockdown. Although there is no direct evidence to clarify the mechanism of chemotherapydriven increases in CDKN1A expression, chemotherapy may inhibit the hypermethylation of the CDKN1A promoter region, which contributes to the rescue of CDKN1A from transcriptional inactivation in breast cancer [26].

CDKN1A is well known for mediating cell cycle arrest in cancer cells [27]; however, the in-depth mechanism of CDKN1A in chemoresistance is unclear, and the role of CDKN1A in the process of chemoresistance is not consistent in different cancers. For instance, the suppressing cell growth and promoting cell cycle arrest functions of CDKN1A were observed in gastric cancer and prostate cancer [27, 28]. In contrast, we found that the expression of CDKN1A in TNBC cells was upregulated with the same trends as those observed for the expression levels of PTN and PTPRZ1 during chemotherapy, which is consistent with previous reports [29]. Moreover, we also demonstrated that increased CDKN1A expression may help TNBC cells escape cell cycle arrest during chemotherapy. It has been shown that upregulated CDKN1A expression regulates cell proliferation in TNBC cells by rescuing the inhibitory effects of liver receptor homolog-1 (LRH-1) in a p53-independent manner [29], which provides the attractive prospect that the CDKN1A/PTN/PTPRZ1 axis could be targeted for the treatment of tumours that are resistant to chemotherapy. However, we only observed the tumour-promoting effects of the CDKN1A-PTN-PTPRZ1 axis in vitro in the present study, and more evidence related to the therapeutic potential of this axis should be confirmed in vitro and in vivo in the future.

The binding of PTN to PTPRZ1 increases the tyrosine phosphorylation of downstream molecules, thus activating the signalling pathways related to cell adhesion, survival and migration [30]. We demonstrated the tumour-promoting effects of PTN in breast cancer cells undergoing chemotherapy involved the upregulation of the expression of PTPRZ1, while the increased expression of PTPRZ1 could also stimulate the secretion of PTN to form a positive feedback loop in TNBC cells. In the present study, we found that the inhibition of rhPTN on Dox could be decreased by siPTPRZ1, and the promotion of siPTN on Dox could be inhibited by overexpressed PTPRZ1. It seems that overexpression of PTPRZ1 has an effect that does not depend on PTN expression. In some other reports, PTN was found not the only activation of PTPRZ1 pathway, inflammation and immune can also activate the PTPRZ1 pathway, such as interleukin (IL)-34, an important receptor of PTPRZ1, promotes the expression of PTPRZ1 in inflammatory bowel disease (IBD) [31]. Whether other factors exist in breast cancer cells that affect the expression of PTPRZ1 under the treatment of Dox, we still need more evidence.

Further investigations of the downstream mechanism revealed that the CDKN1A/PTN/PTPRZ1 axis contributes to chemoresistance by activating the NF- $\mathrm{kB}$ pathway. The NF-kB family is composed of five subunits (p65/RelA, c-Rel, RelB, p105-p50/ NF-kB 1, and p100-p52/ NF-кB 2) and participates in the processes of cell cycling, immunity, angiogenesis, cell adhesion and apoptosis [32, 33]. It is well known that the activation of the NF- $\kappa B$ pathway depends on IKK complex activation and $I \kappa B$ degradation [34]. Our study suggests that Dox could significantly upregulate the expression of IKK $\alpha$, p65 and p50 and induce the degradation of $I K K \beta$ and $\mathrm{I} \kappa \mathrm{B} \alpha$, suggesting that the classical NF- $\kappa \mathrm{B}$ pathway was activated during chemotherapy. In addition, we found that the Dox + PTPRZ1 group showed more obvious activation of the NF- $\mathrm{kB}$ pathway than PTPRZ1 group, thus demonstrating that PTPRZ1 plays an essential role in promoting the activation of the NF- $\mathrm{kB}$ pathway in breast cancer cells undergoing chemotherapy. It has been shown that blocking NF- $\mathrm{kB}$ could restore the sensitivity of breast cancer cells to tamoxifen [35], and the possible mechanism is that the activation of the NF- $\mathrm{kB}$ pathway could dysregulate the apoptotic response by causing the loss of mitochondrial function and death receptor signalling, resulting in transcriptional dysregulation of apoptotic genes [36].

\section{Conclusions}

In conclusion, we have demonstrated that the expression levels of PTN and PTPRZ1 were upregulated in breast cancer tissue after chemotherapy compared to tissue before chemotherapy. High PTN/PTPRZ1 expression is associated with poor chemosensitivity in breast cancer patients. Chemotherapy-driven increases in the 
CDKN1A/PTN/PTPRZ1 axis activate the NF-kB pathway in breast cancer cells. Overall, our results indicate that the chemotherapy-driven increases in the CDKN1A/PTN/PTPRZ1 axis are critical intrinsic factors of therapy-induced chemoresistance in breast cancer and that disrupting the CDKN1A/PTN/PTPRZ1 axis is a novel strategy to enhance the chemosensitivity of breast cancer, especially in the triple-negative subtype.

\section{Abbreviations \\ CDKN1A: Cyclin-dependent kinase inhibitor 1 A; DDP: Cisplatin; Dox: Doxorubicin; ER: Oestrogen receptor; GBM: Glioblastoma multiforme; LRH- 1: Liver receptor homolog-1; NAC: Neoadjuvant chemotherapy; NF-kB: Nuclear factor kB; pCR: Pathologic complete response; PgR: Progesterone receptor; PTN: Pleiotrophin; PTPRZ1: Protein tyrosine phosphatase zeta; TNBC: Triple- negative breast cancer}

\section{Acknowledgements}

We also thank Professor Jun-Li Luo and Professor Shirley Feng for technical assistance.

\section{Funding}

This work was supported by grants from the National Natural Science Foundation of China (81441084), the Natural Science Foundation of the Hunan Province of China (2015jj4059) and the Development and Reform Commission of the Hunan Province of China (2014658)

\section{Availability of data and materials}

The authors declare that all data used in this study are available in the article and Additional files.

\section{Authors' contributions}

Conceived and designed the study: PH and WJY. Development of the methodology: DJOY and MYL. Acquisition of the data: QYL, RZ and QYZ. Analysis and interpretation of the data: LL, JS, PZ and PL. Writing of the manuscript: PH and DJOY. Administrative, technical, or material support: SC and WJY. All authors read and approved the final manuscript.

\section{Ethics approval and consent to participate}

Not applicable.

\section{Consent for publication}

All authors have read and approved the final manuscript and consented to its publication.

\section{Competing interests}

The authors declare that they have no competing interests.

\section{Publisher's Note}

Springer Nature remains neutral with regard to jurisdictional claims in published maps and institutional affiliations.

\section{Author details}

'Department of General Surgery, the Second Xiangya Hospital, Central South University, No.139 Renmin Road, Changsha 410011, China. ²Department of General Surgery, Xiangya Hospital, Central South University, No.87 Xiangya Road, Changsha 410008, China.

Received: 2 August 2018 Accepted: 15 November 2018

Published online: 29 November 2018

\section{References}

1. Siegel RL, Miller KD, Jemal A. Cancer statistics, 2018. CA Cancer J Clin. 2018; 68:7-30.

2. Zou Q, Zhou E, Xu F, Zhang D, Yi W, Yao J. A TP73-AS1/miR-200a/ZEB1 regulating loop promotes breast cancer cell invasion and migration. J Cell Biochem. 2018;119:2189-99.
3. Bianchini G, Balko JM, Mayer IA, Sanders ME, Gianni L. Triple-negative breast cancer: challenges and opportunities of a heterogeneous disease. Nat Rev Clin Oncol. 2016;13:674-90.

4. Zhu QN, Wang G, Guo Y, Peng Y, Zhang R, Deng JL, Li ZX, Zhu YS. LncRNA $\mathrm{H} 19$ is a major mediator of doxorubicin chemoresistance in breast cancer cells through a cullin4A-MDR1 pathway. Oncotarget. 2017:8:91990-2003.

5. Cortazar P, Zhang L, Untch M, Mehta K, Costantino JP, Wolmark N, Bonnefoi H, Cameron D, Gianni L, Valagussa P, et al. Pathological complete response and long-term clinical benefit in breast cancer: the CTNeoBC pooled analysis. Lancet. 2014;384:164-72.

6. Chang Y, Zuka M, Perez-Pinera P, Astudillo A, Mortimer J, Berenson JR, Deuel TF. Secretion of pleiotrophin stimulates breast cancer progression through remodeling of the tumor microenvironment. Proc Natl Acad Sci U S A. 2007;104:10888-93.

7. Fu F, Xiao XI, Zhang T, Zou Q, Chen Z, Pei L, Su J, Yi W. Expression of receptor protein tyrosine phosphatase zeta is a risk factor for triple negative breast cancer relapse. Biomed Rep. 2016:4:167-72.

8. Tang Z, Li C, Kang B, Gao G, Li C, Zhang Z. GEPIA: a web server for cancer and normal gene expression profiling and interactive analyses. Nucleic Acids Res. 2017:45:W98-W102.

9. Huang P, Mao LF, Zhang ZP, Lv WW, Feng XP, Liao HJ, Dong C, Kaluba B, Tang XF, Chang S. Down-regulated miR-125a-5p promotes the reprogramming of glucose metabolism and cell malignancy by increasing levels of CD147 in thyroid Cancer. Thyroid. 2018;28:613-23.

10. Diamantopoulou Z, Kitsou P, Menashi S, Courty J, Katsoris P. Loss of receptor protein tyrosine phosphatase beta/zeta (RPTPbeta/zeta) promotes prostate cancer metastasis. J Biol Chem. 2012;287:40339-49.

11. Deng S, Tang S, Dai C, Zhou Y, Yang X, Li D, Xiao X. P21(Waf1/Cip1) plays a critical role in furazolidone-induced apoptosis in $\mathrm{HepG} 2$ cells through influencing the caspase-3 activation and ROS generation. Food Chem Toxicol. 2016:88:1-12.

12. Forbus J, Spratt H, Wiktorowicz J, Wu Z, Boldogh I, Denner L, Kurosky A, Brasier RC, Luxon B, Brasier AR. Functional analysis of the nuclear proteome of human A549 alveolar epithelial cells by HPLC-high resolution 2-D gel electrophoresis. Proteomics. 2006;6:2656-72.

13. Yu Y, Zhang M, Wang N, Li Q, Yang J, Yan S, He X, Ji G, Miao L. Epigenetic silencing of tumor suppressor gene CDKN1A by oncogenic long noncoding RNA SNHG1 in cholangiocarcinoma. Cell Death Dis. 2018;9:746.

14. Abbas T, Dutta A. p21 in cancer: intricate networks and multiple activities. Nat Rev Cancer. 2009;9:400-14

15. Lee KM, Choi WI, Koh DI, Kim YJ, Jeon BN, Yoon JH, Lee CE, Kim SH, Oh J, Hur MW. The proto-oncoprotein KR-POK represses transcriptional activation of CDKN1A by MIZ-1 through competitive binding. Oncogene. 2017;36:4951.

16. Russo A, Saide A, Cagliani R, Cantile M, Botti G, Russo G. rpL3 promotes the apoptosis of p53 mutated lung cancer cells by down-regulating CBS and NFkappaB upon 5-FU treatment. Sci Rep. 2016;6:38369.

17. Zhou S, Liao L, Chen C, Zeng W, Liu S, Su J, Zhao S, Chen M, Kuang Y, Chen X, Li J. CD147 mediates chemoresistance in breast cancer via ABCG2 by affecting its cellular localization and dimerization. Cancer Lett. 2013;337:285-92.

18. Shi Y, Ping YF, Zhou W, He ZC, Chen C, Bian BS, Zhang L, Chen L, Lan X, Zhang XC, et al. Tumour-associated macrophages secrete pleiotrophin to promote PTPRZ1 signalling in glioblastoma stem cells for tumour growth. Nat Commun. 2017:8:15080.

19. Shang D, Xu X, Wang D, Li Y, Liu Y. Protein tyrosine phosphatase zeta enhances proliferation by increasing beta-catenin nuclear expression in VHLinactive human renal cell carcinoma cells. World J Urol. 2013;31:1547-54.

20. Fang W, Hartmann N, Chow DT, Riegel AT, Wellstein A. Pleiotrophin stimulates fibroblasts and endothelial and epithelial cells and is expressed in human cancer. J Biol Chem. 1992;267:25889-97.

21. Zhang N, Zhong R, Wang ZY, Deuel TF. Human breast cancer growth inhibited in vivo by a dominant negative pleiotrophin mutant. J Biol Chem. 1997:272:16733-6.

22. Perez-Pinera P, Garcia-Suarez O, Menendez-Rodriguez P, Mortimer J, Chang Y, Astudillo A, Deuel TF. The receptor protein tyrosine phosphatase (RPTP) beta/zeta is expressed in different subtypes of human breast cancer. Biochem Biophys Res Commun. 2007;362:5-10.

23. Zins K, Heller G, Mayerhofer M, Schreiber M, Abraham D. Differential prognostic impact of interleukin-34 mRNA expression and infiltrating immune cell composition in intrinsic breast cancer subtypes. Oncotarget. 2018;9:23126-48.

24. Maeda N, Hamanaka H, Shintani T, Nishiwaki T, Noda M. Multiple receptorlike protein tyrosine phosphatases in the form of chondroitin sulfate proteoglycan. FEBS Lett. 1994;354:67-70. 
25. Kuboyama K, Fujikawa A, Suzuki R, Tanga N, Noda M. Role of chondroitin sulfate (CS) modification in the regulation of protein-tyrosine phosphatase receptor type Z (PTPRZ) activity: PLEIOTROPHIN-PTPRZ-A SIGNALING IS INVOLVED IN OLIGODENDROCYTE DIFFERENTIATION. J Biol Chem. 2016;291:18117-28.

26. Askari M, Sobti RC, Nikbakht M, Sharma SC. Aberrant promoter hypermethylation of p21 (WAF1/CIP1) gene and its impact on expression and role of polymorphism in the risk of breast cancer. Mol Cell Biochem. 2013;382:19-26.

27. Liu J, Ben Q, Lu E, He X, Yang X, Ma J, Zhang W, Wang Z, Liu T, Zhang J, Wang $\mathrm{H}$. Long noncoding RNA PANDAR blocks CDKN1A gene transcription by competitive interaction with p53 protein in gastric cancer. Cell Death Dis. 2018:9:168.

28. Zhang Y, Jiang F, He H, Ye J, Mao X, Guo Q, Wu SL, Zhong W, Wu CL, Lin N. Identification of a novel microRNA-mRNA regulatory biomodule in human prostate cancer. Cell Death Dis. 2018;9:301.

29. Bianco S, Jangal M, Garneau D, Gevry N. LRH-1 controls proliferation in breast tumor cells by regulating CDKN1A gene expression. Oncogene. 2015; 34:4509-18.

30. Deuel TF, Zhang N, Yeh HJ, Silos-Santiago I, Wang ZY. Pleiotrophin: a cytokine with diverse functions and a novel signaling pathway. Arch Biochem Biophys. 2002;397:162-71.

31. Zwicker S, Bureik D, Bosma M, Martinez GL, Almer S, Bostrom EA. Receptortype protein-tyrosine phosphatase zeta and Colony stimulating Factor-1 receptor in the intestine: cellular expression and cytokine- and chemokine responses by Interleukin-34 and Colony stimulating Factor-1. PLoS One. 2016;11:00167324.

32. Greten FR, Karin M. The IKK/NF-kappaB activation pathway-a target for prevention and treatment of cancer. Cancer Lett. 2004;206:193-9.

33. Karin M, Cao Y, Greten FR, Li ZW. NF-kappaB in cancer: from innocent bystander to major culprit. Nat Rev Cancer. 2002;2:301-10.

34. Karin M. Nuclear factor-kappaB in cancer development and progression. Nature. 2006;441:431-6.

35. Yde CW, Emdal KB, Guerra B, Lykkesfeldt AE. NFkappaB signaling is important for growth of antiestrogen resistant breast cancer cells. Breast Cancer Res Treat. 2012;135:67-78.

36. Godwin P, Baird AM, Heavey S, Barr MP, O'Byrne K, Gately K. Targeting nuclear factor-kappa B to overcome resistance to chemotherapy. Front Oncol. 2013;3:120

Ready to submit your research? Choose BMC and benefit from:

- fast, convenient online submission

- thorough peer review by experienced researchers in your field

- rapid publication on acceptance

- support for research data, including large and complex data types

- gold Open Access which fosters wider collaboration and increased citations

- maximum visibility for your research: over $100 \mathrm{M}$ website views per year

At $\mathrm{BMC}$, research is always in progress.

Learn more biomedcentral.com/submissions 